\title{
High-Throughput Automated System for Crystallizing Membrane Proteins in Lipidic Mesophases
}

\author{
Avinash Peddi, Lalitha Muthusubramaniam, Yuan F. Zheng, Fellow, IEEE, Vadim Cherezov, \\ Yohann Misquitta, and Martin Caffrey
}

\begin{abstract}
A high-throughput robotic system has been developed to enable the automatic handling of nanoliter volumes of highly viscous biomaterials for crystallizing membrane proteins using lipidic mesophases. The in meso method introduced a few years ago has produced crystal structures of a number of important membrane proteins. The bottleneck to achieve high throughput automation of this, so-called in meso method, is the handling of nanoliter volumes of the highly viscous cubic phase as part of the crystallization process. The cubic phase sticks to everything that it has contact with and has a tendency to disable dispensing robotic tools. In this paper, we discuss the factors that influence the successful and automatic delivery of nanoliter volumes of the cubic phase. A creative cubic-phase-based coordinate measuring mechanism is presented for controlling the dispensing distance of the cubic phase which is critically important for the successful performance of the system. A mathematical model describing the cubic-phase delivery is proposed and verified. We also present the optimization of liquid handling parameters for the successful and automatic delivery of different precipitant solutions. The performance characteristics of the robotic system in terms of accuracy and reproducibility of delivering nano volumes of highly viscous biomaterials and micro volumes of different precipitant solutions are reported.
\end{abstract}

Note to Practitioners-Automatic handling of nanoliter volumes of highly viscous biomaterials is a practically challenging yet important task in research-and-development activities of biology and drug industry, including protein crystallization. The latter, however, is a key step for determining the structure of the proteins. Only when the structure of the proteins is revealed, can one understand the function of the proteins and/or develop drugs to cure various kinds of diseases. This paper uses a practical project, crystallizing membrane proteins using lipidic mesophases, as an example to develop approaches for automatically handling highly viscous biomaterials. This paper presents the factors that influence the successful and automatic delivery of nanoliter volumes of viscous bio-

Manuscript received October 12, 2005; revised March 2, 2006. This paper was recommended for publication by Associate Editor Y. Hayashizaki and Editor D. Meldrum upon evaluation of the reviewers' comments. This work was supported in part by the National Science Foundation under Grant IIS-0308078, in part by the National Institutes of Health under Grant GM61070, and in part by the Science Foundation Ireland under Grant 02-IN1-B266.

A. Peddi is with Tecan US, Durham, NC 27703 USA (e-mail: avinash. peddi@tecan.com).

L. Muthusubramaniam is with Genentech, South San Francisco, CA 94080 USA (e-mail: lalitha07@yahoo.com).

Y. F. Zheng (corresponding author) is with the Department of Electrical and Computer Engineering, The Ohio State University, Columbus, OH 43210 USA and also with Shanghai Jiao Tong University, Shanghai 200240, China (e-mail: zheng@ece.osu.edu).

V. Cherezov is with the Scripps Research Institute, La Jolla, CA 92037 USA (e-mail: vcherezo@scripps.edu).

Y. Misquitta is with the School of Sciences, University of Limerick, Limerick 11111, Ireland (e-mail: yohann.misquitta@gmail.com).

M. Caffrey is with the School of Sciences, University of Limerick, Limerick 11111 Ireland, and also with the Department of Chemistry, The Ohio State University, Columbus, OH 43210 USA (e-mail: martin.caffrey@ul.ie).

Digital Object Identifier 10.1109/TASE.2006.880541 materials and introduces a new coordinate measuring mechanism using the viscous biomaterials for controlling the dispensing distance of the biomaterials, which is critical for the successful delivery of viscous materials. A mathematical model describing the delivery of viscous material is for guiding the design of practical delivering systems. This paper also presents a number of parameters for successful and automatic delivery of different precipitant solutions which are involved in the biomaterial screening activities in drug development and biological research. A simple and effective computer vision approach for measuring the volume of bio-solutions and biomaterials in the nanoliter scale is presented which is verified by the fluorescence intensity measurement of the delivered samples. The performance of the automatic system developed in terms of accuracy and reproducibility of delivering nanovolumes of highly viscous biomaterials is reported which proves that the proposed approach is not only practically useful but also feasible for many applications.

Index Terms-Coordinate measuring, crystallography, high-throughput screening, highly viscous biomaterials, laboratory robotics, membrane proteins, miniaturization, nanovolumes.

\section{INTRODUCTION}

$\mathbf{I}$ $\mathrm{N}$ this proteomics era, one of the greatest challenges within the scientific community is to determine the structure with a view to understand the function of proteins. Generally, X-ray crystallography is the most reliable method for determining the structure of proteins at atomic resolution [1], [2]. For X-ray diffraction studies, the protein of interest must be purified and solubilized in an appropriate medium en route to obtaining well-ordered crystals. However, to obtain diffraction-quality crystals, appropriate physical and chemical conditions have to be provided to support crystal growth. In order to determine the optimum physical and chemical conditions that support the crystal growth, a vast combinatorial space must be explored. Thus, crystal screening involves handling a large number of samples which motivates automating the entire crystallization process. There is also a need to minimize protein usage per trial to reduce cost.

Furthermore, crystallizing membrane proteins is more difficult compared to soluble proteins. This is evident from the fact that to date only $\sim 90$ different membrane protein structures compared to $\sim 20000$ soluble protein structures have been deposited in the Protein Data Bank (http://www.rcsb.org; Membrane Protein Data Bank, http://www.mpdb.ul.ie). There has been significant success in automating the soluble protein crystallization process [3], [12]-[15], and similar techniques can be applied to detergent-solubilized membrane proteins when using standard vapor diffusion or in surfo [5] techniques. Here, we 


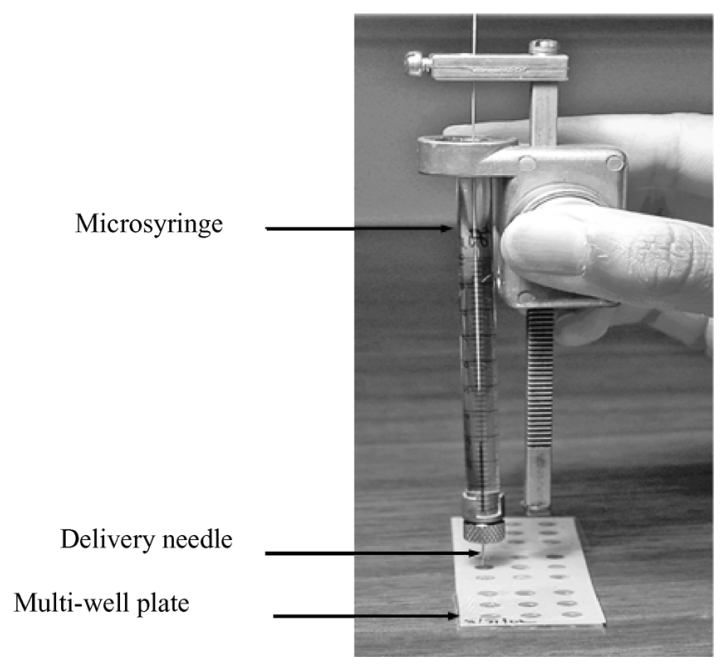

Fig. 1. Cubic-phase delivery in manual mode.

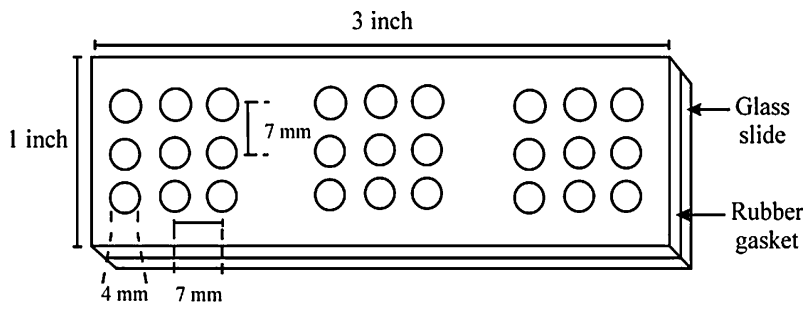

Fig. 2. Twenty-seven-well crystallization plate made from an inert gasket with holes [17].

present a high-throughput automated system for the so-called in meso technique for crystallization of membrane proteins where highly viscous lipid-protein mixtures need to be dispensed as part of the crystallization setup.

Among the available crystallization techniques for membrane proteins [5], the in meso method has been successful in producing crystals used in solving the structures of several important membrane proteins [4], [5]. The crystallization technique can be outlined as follows: 1) mix three parts of lipid (monoolein) with two parts of protein solution/dispersion; 2) overlay it with precipitant solution, and 3) incubate it at a fixed temperature. Given the right conditions of precipitant solution, lipid, detergent, temperature and $\mathrm{pH}$, crystals can form within days to weeks. A highly viscous cubic phase is formed when three parts of monoolein are combined with two parts of bacteriorhodopsin protein solution at room temperature [6]. The delivery of this cubic phase is the most challenging task to automate this crystallization technique.

An example of using the in meso crystallization method that is used in our laboratory is illustrated in Fig. 1. Here, $1 \mu \mathrm{L}$ of precipitant solution is dispensed in a set of wells (Fig. 2) followed by $70 \mathrm{~nL}$ of cubic phase delivered by means of a microsyringe dispenser [22]. The cubic-phase-loaded wells are capped with a glass cover slip and then incubated in a temperature-controlled incubation chamber. The wells are examined on one, three, seven, 14, and 21 days after the crystallization setup to monitor crystal growth. The limitations of setting up crystallization trials in manual mode are throughput, irregularities of delivered sample volumes, and the demand for human dexterity.
An extensive search of the literature failed to uncover any significant work on the automatic and accurate handling of nanoliter volumes of viscous materials in the field of automation science and engineering. We only found some remotely related works. For example, Tokumoto et al. developed a deformation model for viscoelastic materials [7], [8]. The purpose is to automatically shape an object, such as pizza, from viscoelastic materials by using a robot and by treating the materials as lattice with four kinds of viscoelastic elements connecting points of mass. Chen and Zheng proposed a deformation transition graphs approach to guide a robot in shaping deformable objects [9]. Both works do not deal with viscous materials of liquid-crystal type and are not truly relevant to biology. Various research groups studied the handling of soluble proteins where viscosity was not an issue [3], [10]-[15]. A novel microfludic technology has been used for protein structure determination using X-ray crystallography [21]. Meldrum et al. developed automated systems for genome analysis applications which do not require the automated handling of nanovolumes of highly viscous biomaterials [16]. Hence, a robotic system has been developed here at The Ohio State University to address the requirements of high-throughput, automation, miniaturization, and delivery of a highly viscous biomaterial [20].

A high-throughput robotic system that delivers nanovolumes of a highly viscous mesophase and microvolumes of different precipitant solutions to automate the crystallization of membrane proteins was developed and is described in this paper. A detailed description of system hardware, developed by integrating different commercially available components, is presented in Section II. The procedure for calibration and optimization of liquid handling parameters of tips to attain successful delivery of different precipitant solutions is reported in Section III. In Section IV, we describe the newly developed approach for delivery of nanovolumes of cubic phase and key factors guaranteeing the successful delivery of the nanovolume of cubic phase. In Section V, we describe a cubic-phase-based coordinate mechanism which makes use of the conductivity of the cubic phase. The purpose is for online measurement of the height of the glass slides which is closely related to the dispensing distance of viscous materials. The dispensing distance, on the other hand, is a critical factor for the successful delivery of the cubic phase. The mathematical model of cubic phase delivery is presented in Section VI followed by experimental results in Section VII. In this section, the robotic system performance, in terms of accuracy and reproducibility in delivering microvolumes of different precipitant solutions and nano volumes of cubic phase, is presented. The flexibility of the robotic system in setting up different crystallization trials is also provided in the Results section followed by the Conclusion.

\section{HARDWARE OF THE IN MESO ROBOTIC SYSTEM}

This is a robotic system developed to automate the crystallization of membrane and soluble proteins using the in meso technique. The robot was built by integrating Xantus, a commercial liquid handling robotic system (SIAS AG, Hombrechtikon, Switzerland) with a positive displacement microprocessor-controlled microsyringe pump (UMP II with Micro4 controller, World Precision Instruments, Sarasota, FL) 


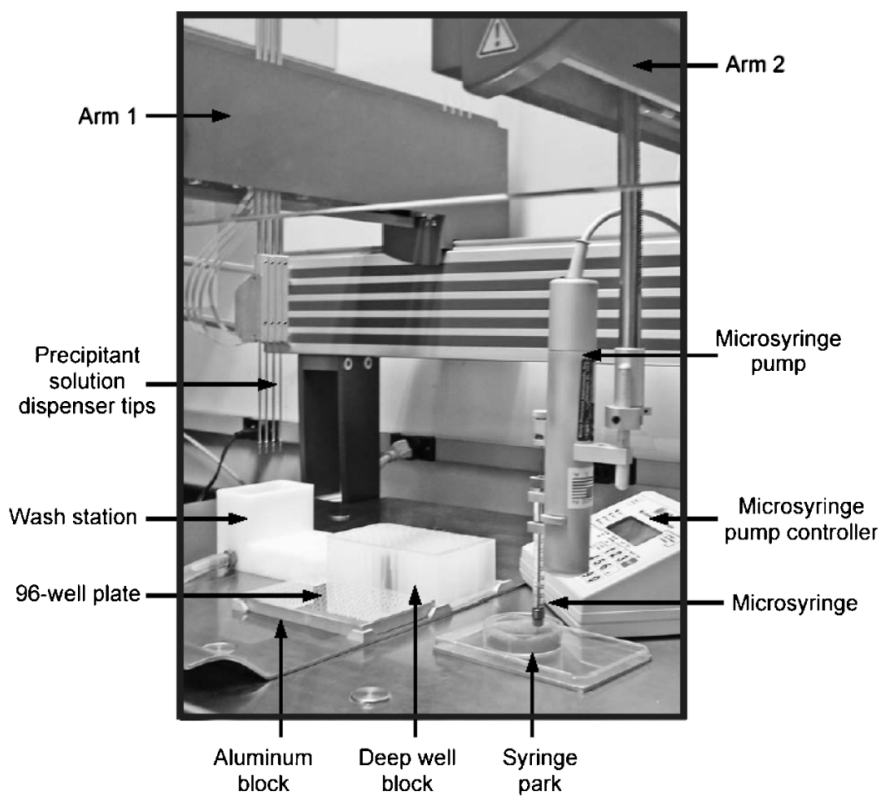

Fig. 3. In meso robotic system.

as shown in Fig. 3. The standard Xantus system has a $1 \times 0.7-\mathrm{m}$ deck with two arms, each of which has three degrees of freedom with linear motion in $X, Y$, and $Z$ directions, respectively. In the standard Xantus robot, Arm 1 is used to aspirate and dispense solutions and Arm 2 has a plate gripper to move plates. The step size of $X, Y$, and $Z$ motors of both arms in the standard system is $100 \mu \mathrm{m}$.

The functionality of Arm 1 for handling solutions is retained in the in meso robotic system. The standard tips are replaced with low-volume tips to aspirate and dispense microliter volumes of precipitant solutions. Arm 1 has four low-volume liquid handlers. The functionality of Arm 2 has been modified to deliver nanoliter volumes of highly viscous mesophase. It is equipped with a positive displacement microprocessor-controlled microsyringe pump which is integrated to Xantus through an input/output (I/O) board to handle protein/lipidic mesophase. The step size of the Arm $2 Z$ motor was changed from 100 to $12.5 \mu \mathrm{m}$ as the dispensing distance (the height of the dispensing needle tip above the base of the well) is a critical factor for the successful delivery of mesophase. Reducing the step size of the $Z$ motor is for the benefit of fine-tuning the dispensing distance, for which the details will be discussed later in this paper.

The positive displacement microprocessor-controlled microsyringe pump's working principle is simple and can handle syringes from $10 \mu \mathrm{L}$ to $1 \mathrm{~mL}$. The volume delivered per step for a particular syringe is constant since the step size of the pump is constant which is $3.189 \mu \mathrm{m}$. Accordingly, for the standard Hamilton gas-tight $100-\mu \mathrm{L}$ syringe used in our laboratory (with a syringe scale length of $60 \mathrm{~mm}$ ), the volume per step is $5.315 \mathrm{~nL} / \mathrm{step}$. The desired volume is delivered by moving the plunger through a fixed number of steps. The actual volume dispensed will be the product of volume per step and the number of steps. Thus, for a desired volume of $50 \mathrm{~nL}$ using a standard $100-\mu \mathrm{L}$ syringe, $47.83 \mathrm{~nL}$ is the volume actually delivered when the plunger is moved by nine steps. Sixteen

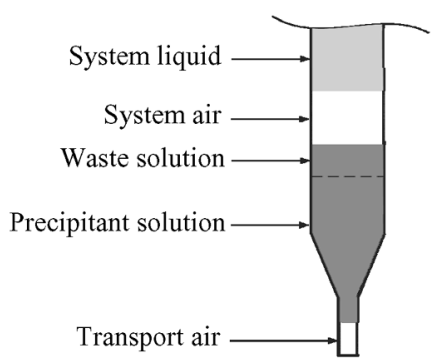

Fig. 4. Schematic of the in meso robotic system tip.

steps would give $85.04 \mathrm{~nL}$. The desired volume and rate of delivery can be adjusted using Micro4, the microsyringe pump controller. The standard delivery rate used in the laboratory for dispensing $50-\mathrm{nL}$ cubic phase from a $100-\mu \mathrm{L}$ standard syringe is $500 \mathrm{~nL} / \mathrm{s}$. This setup is integrated to the Xantus robotic system through an I/O board which generates a $5-\mathrm{V}$ signal on sending a command to deliver a given volume.

\section{DELIVERY OF Microliter Volumes OF PRECIPITANT SOLUTIONS}

One of the objectives of the in meso robotic system we developed is to deliver defined volumes of different precipitant solutions accurately and reproducibly. For this purpose, the liquid handlers are first calibrated for both the aspiration and dispensing of water to the desired accuracy. Then, the liquid handling parameters, such as aspiration speed, waste volume, etc., are optimized for reproducible delivery of different solutions.

Each liquid handler is associated with a separate pump and these pumps are not equalized in terms of their performance. That is, different pumps may dispense different volumes of liquid even with an identical command input. Hence, individual liquid handlers have to be calibrated to determine the specific input corresponding to a particular dispense volume. In our laboratory, the liquid handlers are calibrated for both aspiration and dispensing of 1,10 , and $100 \mu \mathrm{L}$ of solution using a gravimetric technique. One-microliter calibration is required as it is the typical volume of precipitant solutions used for setting up crystallization trials. The tips are also calibrated for $10 \mu \mathrm{L}$ and $100 \mu \mathrm{L}$ as these are nominal volumes used while preparing precipitant solutions during the optimization procedure. For calibrating the tips, 100 or $10 \mu \mathrm{L}$ or ten times $1 \mu \mathrm{L}$ of water is delivered into small, lightweight (500-mg) plastic tubes and weighed using a microbalance (AX205, Mettler-Toledo Inc., Columbus, $\mathrm{OH})$. The volume is converted to weight using a density of $0.99823 \mathrm{~g} \mathrm{ml}^{-1}(293 \mathrm{~K}$, [23]). Based on the average volume of five deliveries, the calibration factor is adjusted to attain the desired target volume. This procedure is repeated until the dispensed or aspirated volume is within 5\% of the nominal volume. A similar procedure is followed for calibrating the liquid handlers to aspirate 1,10 , and $100 \mu \mathrm{L}$ of water.

After calibrating the tips, the liquid handling parameters have to be optimized for delivering different precipitant solutions, as the accuracy and the reproducibility of delivering solutions depends on various parameters, such as aspiration and dispensing speed, system air, transport air, waste volume, and aspiration 


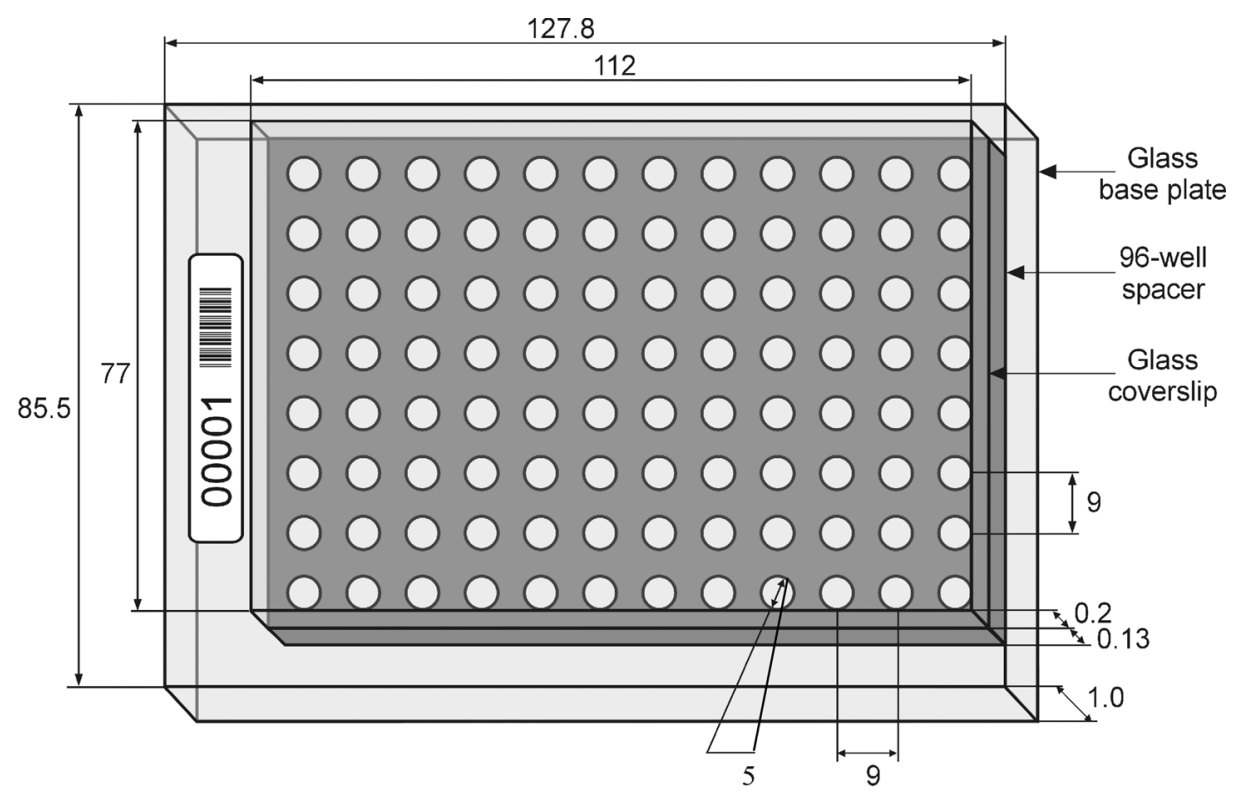

Fig. 5. Schematic of 96-well glass plate with dimensions in millimeters.

and dispensing delay. These liquid handling parameters are described in Fig. 4. For example, when aspirating viscous solutions, there is a tendency for air to be aspirated instead of solutions if speed is too high. So the aspiration speed should be low with a longer post-aspiration delay to attain the desired accuracy. Similarly, dispensing speed should be lowered to avoid the delivery of air instead of solutions. Furthermore, the set of optimal liquid handling parameters differs for different solutions as properties, such as viscosity, surface tension, etc., vary from one solution to another. Thus, the challenging task is to determine a single set of parameters that are optimal for handling a wide range of solutions used in a typical protein crystallization screen.

To address this optimization problem, the liquid handling parameters that affect the delivery of solutions significantly are determined first. This is performed by delivering $1 \mu \mathrm{L}$ of water and $25 \%(\mathrm{w} / \mathrm{w}$ ) Polyethyleneglycol 4000 (PEG4000) solution in a homemade plate (Fig. 5). This plate is prepared using a flat glass plate, a 96-hole perforated polymer spacer and a glass cover slip. The flat glass plate (Erie Scientific, Portsmouth, NH) has dimensions of $127.8 \times 85.6 \mathrm{~mm}^{2}$ that conforms to the Society for Biomolecular Sciences (SBS) standard with 1-mm thickness. The spacer (Saunders East, Lombard, IL) is a doublestick polyester film that has a footprint of $112 \times 77 \times 0.13 \mathrm{~mm}^{3}$ with 96 holes, each $5 \mathrm{~mm}$ in diameter and separated by $9 \mathrm{~mm}$. The glass-cover slip (Erie Scientific, Portsmouth, $\mathrm{NH}$ ) is made from glass with $112 \times 77 \times 0.2-\mathrm{mm}^{3}$ dimensions. The spacer is placed on the flat glass base plate which creates 96 isolated wells. This is covered by the glass cover slip after loading it completely. As the well thickness is $0.13 \mathrm{~mm}$, the $1 \mu \mathrm{L}$ of dispensed solution in the plate is sandwiched between and in contact with the upper and lower glass surface.

The delivery in each well is evaluated using an imaging system which was developed in our laboratory [20] and automated image analysis software. This automated image analysis software is developed by writing plug-ins using ImageJ
(Wayne Rasband, NIH, Bethesda, MD, USA; available free from http://rsb.info.nih.gov/ij/) and Adobe Photoshop (Adobe Systems, Inc., San Jose, CA). The automated image analysis determines the area occupied by the delivered solution and the volume of delivery is calculated as the product of area and well spacer thickness $(0.13 \mathrm{~mm})$. Water and 25\%(w/w) PEG4000 are used to perform the analysis as they cover the range of viscosities likely to be encountered in a typical screen.

From the initial optimization experiments, it was determined that the aspiration and dispensing speed $(50-300 \mu \mathrm{L} / \mathrm{s})$, waste volume $(0-2 \mu \mathrm{L})$, and system air $(0-10 \mu \mathrm{L})$ significantly affect the delivery of different solutions. Therefore, these parameters were fine-tuned to determine the optimum configuration set. For each parameter, the delivery is evaluated using the previously mentioned automated image analysis. From the measurements, it is determined that the optimum configuration set includes aspiration and dispense speed of $50 \mu \mathrm{L} / \mathrm{s}, 1-\mu \mathrm{L}$ waste volume, $5-\mu \mathrm{L}$ system air gap, 0 -s aspiration and dispensing delays, and 0 $\mu \mathrm{L}$ of transport air. The performance of the system in delivering precipitant solutions is presented in the Results section.

\section{Delivery of Nanovolumes of Cubic Phase}

It is important to automate the delivery of lipid/protein mesophase so as to automate the in meso crystallization technique. Furthermore, there is a strong need to scale down the consumption of material with a view to minimize the protein usage per trial.

Automating the delivery of the cubic phase is achieved by using a microprocessor-controlled positive displacement microsyringe pump and motors for precisely positioning the needle tip above the base of the well. Since automatic and accurate delivery of highly viscous biomaterials has never been seriously studied in automation science and engineering, it is necessary to take a close look at the delivery process to determine the factors that impact the dispensing of nanoliter volumes of highly viscous biomaterials. The viscosity of the 


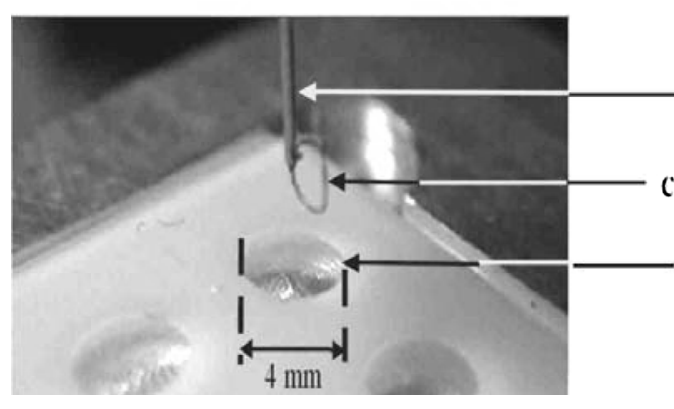

(a)

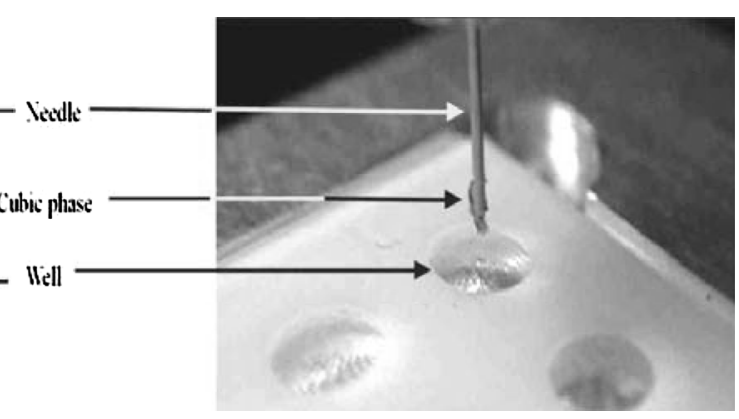

(b)

Fig. 6. (a) Cubic phase forms a loop and comes back toward the needle when it is too far from the bottom of the well during delivery. (b) Cubic phase remains stuck to the needle when it is too close to the bottom of the well during delivery.

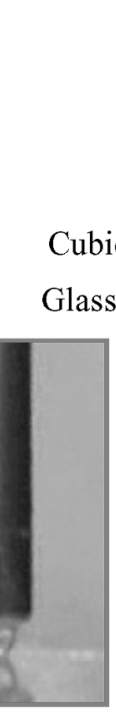

A)

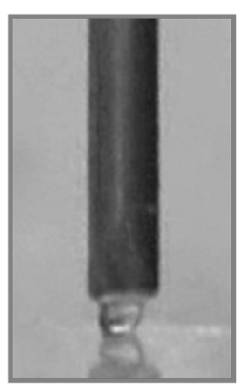

(A)

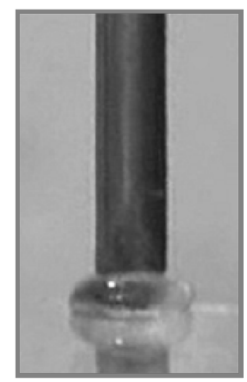

$0.2 \mathrm{~s}$

(B)
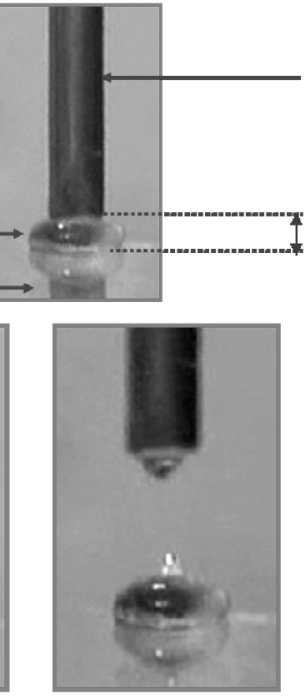

$0.9 \mathrm{~s}$

(C)

Needle

( $0.46 \mathrm{~mm}$ outer diameter)

$0.3 \mathrm{~mm}$

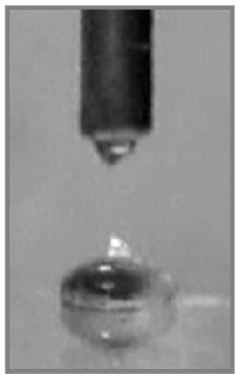

$1 \mathrm{~s}$

(D)

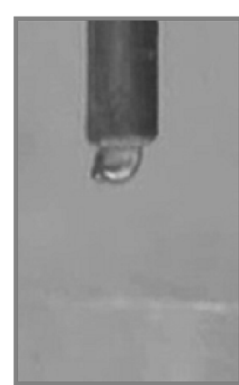

$1.7 \mathrm{~s}$

(E)

Fig. 7. Sequence of events occurring during the delivery of cubic phase. $100 \mathrm{~nL}$ of cubic phase (Monoolein/Methylene blue solution, $63 / 37$ (w/w)) delivered from $0.3 \mathrm{~mm}$ above the base of the well.

cubic phase has not been determined quantitatively in our work, but it has been qualitatively determined to be higher than $3000 \mathrm{cP}$ which is the viscosity of honey (http://www.sheepscotmachine.com/viscosity.html).

The cubic-phase dispense has been observed using needles of different sizes and while dispensing them from different heights. The experimental observations indicate that dispensing distance, texture, and volume of material to be delivered, and the diameter and tip uniformity of needle impact the delivery of nanoliter volumes of highly viscous biomaterials. Of these, the most important factor is the dispensing distance (i.e., the height of the dispensing needle tip above the base of the well). The importance of the dispensing distance is clearly demonstrated from the following observation [Fig. 6(a) and (b)].

If the dispensing tool is too far away from the base of the well, the cubic phase emerging from the needle forms a continuous tube that loops back to the needle and, hence, is not delivered to the well [Fig. 6(a)]. Similarly, if the dispensing needle is too close to the base of well, some of the delivered cubic phase balls up and sticks to the needle [Fig. 6(b)]. Thus, a part or all of the delivered cubic phase is transferred to the next well resulting in an unsuccessful delivery. Hence, it is very important to precisely position the dispensing tool at the right distance from the base of the well.

The time-lapsed panels in Fig. 7 show the sequence of events that occur during the nanoliter-scale viscous material dispensing process. In A, the syringe dispenser together with dispensing needle and residual from the previous trial is at the desired height above the base of the well. In B, the desired volume of cubic phase is expelled. In $\mathrm{C}$, the cubic-phase dispenser is retracted back but there is a small carryover volume at the needle tip, accompanied by the formation of a break point. In D, one can notice the increase in the carryover volume at the needle tip. When no force is being exerted, the relaxation of the viscous material results in a slow flow of cubic phase, which accounts for the increase in the carryover at the needle tip. In E, the needle tip is shown with the complete final carryover volume before going to the next well.

From Fig. 7, one can see that the carryover volume affects the accurate dispense of nanovolumes of highly viscous bioma- 


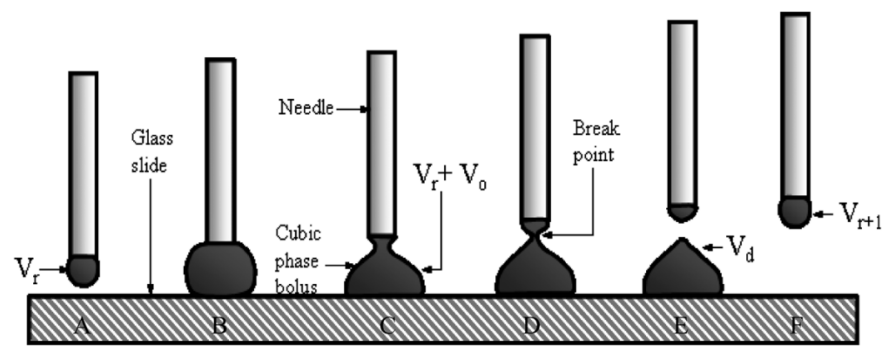

Fig. 8. Cartoon representing the sequence of events during the delivery of viscous biomaterials.

terials. The carryover volume is, however, determined by two factors. One is where the break point is and the other is how the viscous material relaxes from the needle. These two factors need to be carefully studied.

\section{A. Break Point Carryover Volume}

The formation of a break point is explained in Fig. 8, which represents the events observed during the delivery of the viscous mesophase. In A, the syringe dispenser, together with the needle, is at the adequate height above the base of the well with the carryover from the previous trial. In B, the desired volume of the cubic phase is expelled. In $\mathrm{C}$, the cubic phase dispenser is retracted back and, thus, the shape of cubic phase changes because both the needle and well stick to the mesophase. In D, one can observe the formation of a break point which governs the amount of cubic phase being delivered to the well. In E, the needle moves back up with the break point carryover volume. In $\mathrm{F}$, we see the needle with carryover volume before going on to the next well. The increase in the carryover volume from $\mathrm{E}$ to $\mathrm{F}$ at the needle tip is due to relaxation of the syringe system as discussed below.

\section{B. Relaxation of the Viscous Biomaterial}

During the delivery of the viscous biomaterial, the syringe plunger moves to expel its contents. Due to the high viscosity of the dispensing material, there is strong resistance to flow; as a result, pressure builds up in the needle which causes the wall to deflect. The system relaxes only when the cubic phase is expelled from the needle. The time constant of the relaxation and the amount of material expelled during relaxation depends on the viscosity of the dispensed material and the diameter of the dispensing needle. It has been observed that under typical dispensing conditions, the relaxation time could be as long as several seconds. This cannot be tolerated in a high-throughput application. However, if the delivery time per well is more than the time for complete expulsion of the viscous material, then all of the wells excepting the first few ones, receive the desired volume as they reach steady state. To this end, the syringe is primed by expelling a small volume of material before the delivery into the first well so as to avoid the pre-steady state underfilling of the first few wells.

\section{Cubic-Phase-Based Coordinates Measuring OF DisPENSING DisTANCE}

As noted, the dispensing distance is critically important for the successful delivery of the cubic phase. It is determined by the height of the well base (i.e., the thickness of the glass slide) (see Fig. 2) when the position of the dispensing tip is determined. Our measurements show that individual slides are uniformly thick to the extent that a single measurement along each slide would suffice as a reference for all the wells on a given slide. However, the variability between slides is significantly large. Commercially available slides that we have worked with come with a thickness specification of $1 \mathrm{~mm}$. We have measured the thickness separately and found that within slides, the variation of the thickness is between $\pm 10 \mu \mathrm{m}$. Between slides within a lot, the thickness is $1 \mathrm{~mm} \pm 50 \mu \mathrm{m}$ (for 8 slides in each lot). Between three lots, the variation of the thickness was again found to be $\pm 50 \mu \mathrm{m}$ for a total of 24 slides. This variation is too large for our current application which requires that we know the thickness down to $10 \mu \mathrm{m}$ for reproducible delivery of the cubic phase. Also, the needle must move down at a high speed for the purpose of efficiency. But during this time, the needle may impact the slide at a high speed and might get bent or break the slide when the thickness of the slide is not known.

For the two reasons just mentioned, the position of each slide must be measured individually. Ideally, we want to do this automatically and in real time. We propose an innovative solution which uses the cubic phase dispensed by the robot itself as a transducer for measuring the coordinates of the glass slide.

The idea of using the cubic phase as a transducer is based on the fact that it contains almost 50\% water [6] and, hence, conducts electricity, although its resistance is high. We use this latter property to detect contact. The advantage of this approach is that coordinate measurement is simple, cost-effective, and sensitive and is also an integral part of the system. The working of the cubic-phase sensor is described below.

A metal clamp is used to fix the glass slide onto the $X Y$ translation stage. As the tip of the delivery syringe drives down and contacts this clamp, the resistance between the closed loop formed by the metallic needle tip and the clamp becomes zero (Fig. 9). This position is then recorded as the reference position for the glass slide. However, a practical difficulty with this approach is that as the needle is moving down at a high speed, it is possible that it collides and crashes with the clamp. To avoid this problem, we expel a small bolus of cubic phase at the needle tip. When this bolus makes contact with the clamp, we can read an intermediate value of resistance around the loop formed by the cubic-phase buffer, the needle tip, and the clamp. When the resistance drops from a high open-loop value to this intermediate value, a signal can be sent to alert the robot that the needle tip and the clamp are in close proximity. The rate of downward travel of the needle tip is then reduced significantly and it continues to move down at this lower speed until it makes contact with the clamp.

As explained before, we should be able to identify three distinct zones in the working of the sensor: 1) no-contact, where the needle tip is far away from the clamp, 2) precontact, where the cubic-phase bolus has established contact with the clamp, and 3) contact, where the needle has contacted the clamp surface. There is a signature value of resistance associated with each zone. An electronic circuit was designed to convert this resistance value to a voltage inversely, which could then serve as a signal to alert the robot. 


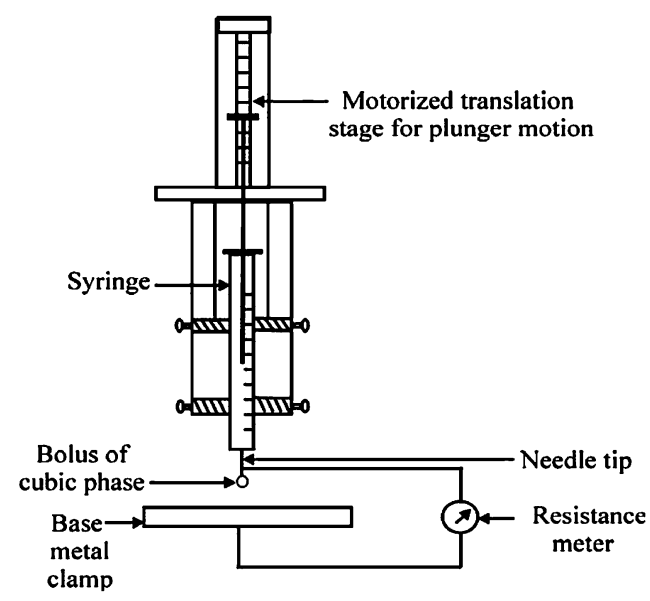

Fig. 9. Cubic-phase-based coordinates measuring machine (CMM).

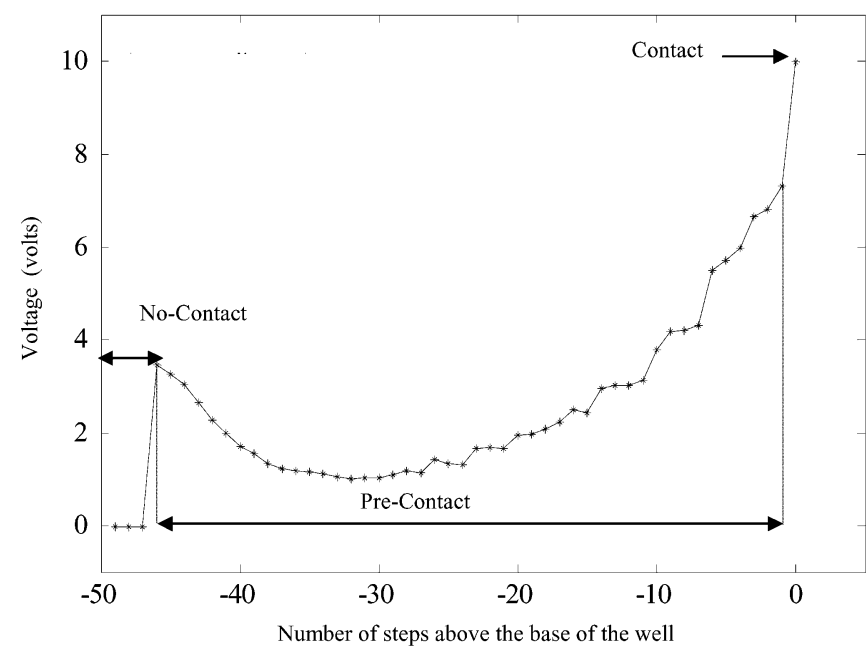

Fig. 10. Cubic-phase-based coordinate measurement-the measured voltage across the reference resistance with $50-\mathrm{nL}$ cubic phase (myverol/methylene blue) at the needle tip as the needle moves toward the metal clamp. Each step represents $12.5 \mu \mathrm{m}$.

Fig. 10 illustrates the results of a study that were conducted using $50 \mathrm{~nL}$ of a cubic-phase sample formed using the lipid, myverol, and a solution of methylene blue. From these data, it is easy to see that the three working zones are clearly distinct from each other and can easily be differentiated. For the circuit we developed, we consider the needle to be in the no-contact (zone 1) when the voltage is less than $0.046 \mathrm{~V}$. When it is greater than $9.8 \mathrm{~V}$, the needle is in the contact (zone 3), and when it is between 0.046 and $9.8 \mathrm{~V}$, the needle is in the precontact state (zone 2).

As mentioned earlier, the step size of Arm 2 in the $\mathrm{Z}$ direction is $12.5 \mu \mathrm{m}$ and, hence, this is the resolution of this coordinate measuring mechanism. That resolution is close to the desired accuracy of $10 \mu \mathrm{m}$ for successful delivery of the cubic phase. The effectiveness and practicality of this method, however, is far more optimal when compared to a separate coordinate measuring machine (CMM).

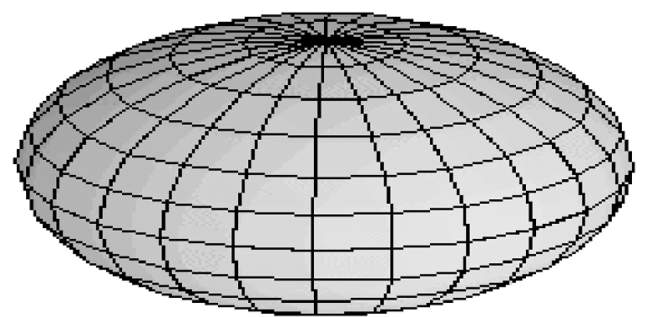

Fig. 11. Ellipsoidal model to describe the shape of the dispensed cubic phase.

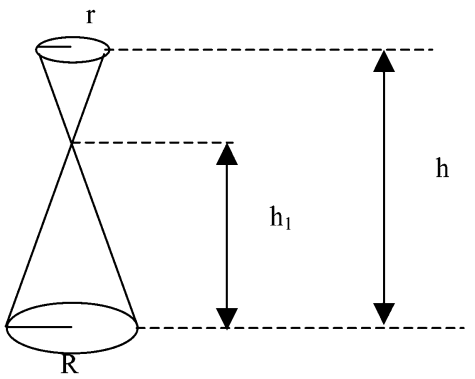

Fig. 12. Conical model to determine break-point position.

\section{Mathematical Modeling}

From the observed set of events that take place during the actual mesophase delivery, we have developed a mathematical model to determine the actual volume delivered to the well. With this model, the volume of material that has to be expelled during priming can also be determined. We provide the mathematical model of two important aspects in the delivery process: the shape of the delivered cubic phase and the formation of the break point.

An ellipsoidal model, as in Fig. 11, is proposed to describe the shape of the delivered mesophase. The length of the major axis represents the diameter of the expelled biomaterial and the minor axis represents the height of the needle tip above the base of the well from which biomaterial is delivered. Thus, the volume of the delivered cubic phase is provided by (1). From (1), it is easy to determine the radius of dispensed cubic phase when the volume and the height from which the material is delivered are known. The radius will determine the area of the footprint where the viscous material is in contact with the bottom of the well. The area will further determine the actual volume that is delivered to the well. The analysis of the latter will be shown.

Once the cubic phase is in contact with the bottom, a conical model is proposed to determine the height above the base of well at which a break point will form as shown in Figs. 8 and 12. By a simple geometry, (2) can be obtained. As mentioned before, the radius of the dispensed cubic phase can be calculated using (1). The position of the break point can be determined using (2) as the inner radius of the dispensing needle, dispensing height, and the radius of the dispensed cubic phase are known

where

$$
V=\frac{4}{3} \pi R^{2} h
$$

$V \quad$ volume of cubic phase expelled;

$R \quad$ radius of the cubic phase at the base of the well;

$V \quad$ height of the needle tip above the well base.

$$
\frac{R}{r}=\frac{h_{1}}{h-h_{1}}
$$


TABLE I

ACCURACY AND COEFFICIENT OF VARIANCE IN DELIVERING $1 \mu \mathrm{L}$ OF WATER AND 25\% PEG4000

\begin{tabular}{|c|c|c|c|c|}
\hline \multirow{2}{*}{ Tip } & \multicolumn{2}{|c|}{ Water } & \multicolumn{2}{c|}{ 25\% PEG 4000 } \\
\cline { 2 - 5 } & Volume $(\mu \mathrm{L})$ & CV & Volume $(\mu \mathrm{L})$ & CV \\
\hline 1 & 0.98 & 7.2 & 0.93 & 7.9 \\
\hline 2 & 1.08 & 7.1 & 1.04 & 7.2 \\
\hline 3 & 0.95 & 11.3 & 0.96 & 11.1 \\
\hline 4 & 1.03 & 6.2 & 1.01 & 6.5 \\
\hline
\end{tabular}

where

$R \quad$ radius of the cubic phase at the base of the well;

$h$ height of the needle tip above the base of the well;

$h_{1} \quad$ height of break point from the well base;

$r \quad$ radius of the needle tip.

An equation that governs the volume delivered (3) to a well for a given height from which the cubic phase is delivered, the amount of volume expelled from the syringe, and the radius of the dispensing needle were developed from (1) and (2). The equations governing the break-point residual volume and the amount of prime volume to be delivered initially are given by (4) and (5), respectively. Verification of the proposed model is presented in the Results section

$$
V_{\mathrm{d}}=\left(V_{\mathrm{o}}+V_{\mathrm{r}}\right)\left(\frac{R^{3}}{R^{3}+r^{3}}\right)
$$

where

$$
\begin{array}{ll}
V_{\mathrm{d}} & \text { volume delivered; } \\
V_{\mathrm{o}} & \text { volume expelled from the syringe; } \\
V_{\mathrm{r}} & \text { break-point carryover volume from the previous well; }
\end{array}
$$

$R \quad$ radius of the cubic phase at the base of the well;

$r \quad$ radius of the needle tip;

$$
V_{\mathrm{r}+1}=\left(V_{\mathrm{o}}+V_{\mathrm{r}}\right)\left(\frac{r^{3}}{R^{3}+r^{3}}\right)
$$

where

$V_{\mathrm{r}+1}$ break-point carryover volume;

$V_{\mathrm{o}} \quad$ volume expelled from the syringe;

$V_{\mathrm{r}}$ break-point carryover phase at the base of the well;

$R \quad$ radius of the cubic phase at the base of the well;

$r \quad$ radius of the needle tip;

$$
V_{\mathrm{p}}=V_{\mathrm{o}}\left(\frac{r^{3}}{R^{3}+r^{3}}\right)
$$

where

$$
\begin{array}{ll}
V_{\mathrm{p}} & \text { initial prime volume; } \\
V_{\mathrm{o}} & \text { volume expelled from the syringe; } \\
R & \text { radius of the cubic phase; } \\
r & \text { radius of the needle tip. }
\end{array}
$$

\section{RESULTS}

\section{A. System Validation}

The performance of the system in delivering nanoliter volumes of mesophase and microliter volumes of different precip- itant solutions is evaluated. This quantitative analysis is critical to ensure proper system functionality in setting up crystallization trials. The detailed procedure and the results of quantitative analysis of different solutions and mesophase delivery are presented in the following subsections.

Delivery of Microliter Volumes of Precipitant Solutions: The accuracy and precision of the in meso robotic system in delivering microliter volumes of precipitant solutions is performed using image analysis. One microliter of water is dispensed into the first four wells of the 96-well crystallization plate by four low-volume tips. This is followed by the delivery of $1 \mu \mathrm{L}$ of 25 $\%$ PEG4000 solution in the next four wells of the first column. Similarly, all 12 columns of the 96-well crystallization plate are loaded with $1 \mu \mathrm{L}$ of water and 25\% PEG4000. Thus, each plate consists of 12 replicates of a particular solution delivered by a specific tip. The plate is then covered with a cover slip such that the dispensed solution is sandwiched between the glass slides. The plate is scanned immediately using the imaging system mentioned earlier. The images are analyzed automatically using the image analysis protocol. This automated image analysis determines the area of the solution in each well. The volume of the delivered solution is obtained from the product of the calculated area and the well thickness $(130 \mu \mathrm{m})$.

Using the optimal liquid handling parameter configuration described in Section III, the accuracy and reproducibility of the robotic system in delivering water and a 25\% PEG4000 solution are presented in Table I. The results are obtained from more than 50 dispenses with each tip. The metrics used to quantify the performance of liquid handlers are the average and coefficient of variance $(\mathrm{CV})$ of replicates. The $\mathrm{CV}$ is defined as the ratio of the standard deviation to the sample average. The results indicate that the $\mathrm{CV}$ for delivering water and 25\% PEG4000 is less than $8 \%$ except for tip 3, which is about $11.3 \%$ : slightly higher than the desired $10 \%$. We attribute this larger than normal CV to the higher variation in the output of the pump associated with tip 3.

Delivery of Nanoliter Volumes of Highly Viscous Cubic Phase: The unique capability of the robotic system is to deliver nanoliter volumes of highly viscous mesophase. The performance is measured using direct image analysis as described in the previous subsection. The given volume (50 or $20 \mathrm{~nL})$ of cubic phase is delivered in the 96-well crystallization plate and then the plate is covered with a cover slip. The entire delivery process is conducted in a humidified atmosphere to ensure that there is no dehydration effect. The dispensed cubic-phase bolus has a relatively uniform disc shape after being sandwiched between the glass slides. A sample plate loaded with $100 \mathrm{~nL}$ of the cubic phase (Monoolein/Bis-CarboxyEthyl-Carboxy-Fluorescein (BCECF) solution 60/40 (w/w)) dispensed 23 steps $(287.5 \mu \mathrm{m})$ above the base of the well and is shown in Fig. 13. 


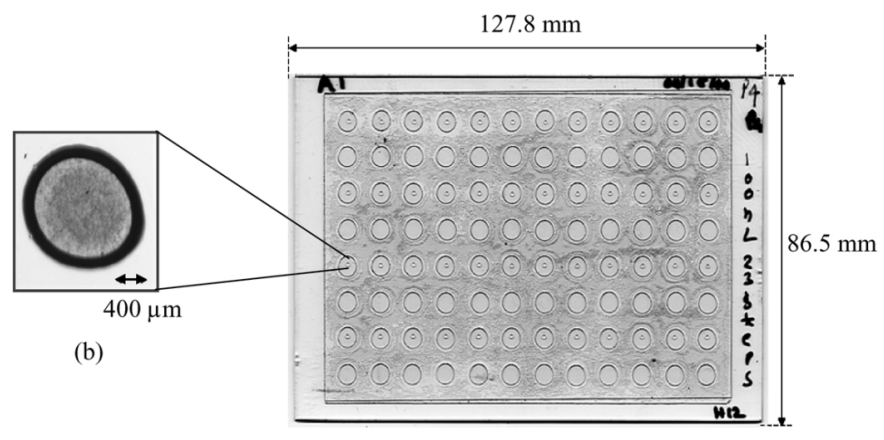

(a)

Fig. 13. (a) Sample plate produced by the in meso robotic system delivering $100 \mathrm{~nL}$ of cubic phase (Monoolein/BCECF solution 60/40(w/w)) from 23 steps above the base of the well. (b) Zoom-in picture of a particular specific well showing the black outline of the cubic-phase bolus.

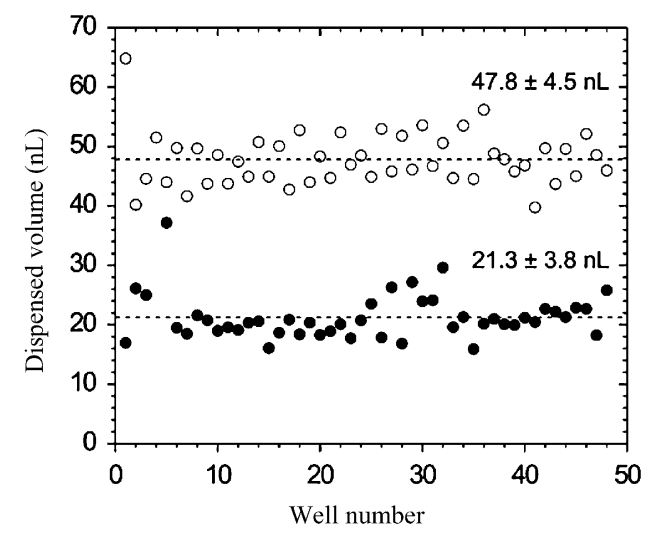

Fig. 14. Accuracy and reproducibility in delivering 20 and $50 \mathrm{~nL}$ of cubic phase.

The accuracy and reproducibility of the system in delivering 50 and $20 \mathrm{~nL}$ of the cubic phase are shown in Fig. 14. The results indicate that the reproducibility of the system in delivering $47.83 \mathrm{~nL}$ is less than $10 \%$ compared to $18 \%$ for delivering 21.26 $\mathrm{nL}$. The accuracy is calculated by measuring the volume dispensed after 100 deliveries. The actual volume for 100 deliveries is calculated from the displacement of the syringe plunger. From this experimental result, $50 \mathrm{~nL}(47.83 \mathrm{~nL})$ is chosen as the standard volume in setting up crystallization trials. This is due to the fact that the reproducibility in delivering $50 \mathrm{~nL}$ is much higher compared to that of $20 \mathrm{~nL}(21.26 \mathrm{~nL})$. Furthermore, working with larger volume reduces the effect of dehydration and has a somewhat higher probability of crystallization and generally produces bigger crystals [17].

\section{B. Mathematical Model Verification}

The proposed mathematical model is verified using the same experiments that are used to determine the accuracy and reproducibility of cubic-phase delivery. A set of 48 wells (alternate rows in the entire 96-well plate) are delivered with a particular composition of the cubic phase from a given dispensing distance. The volume of the delivered cubic phase in each bolus is calculated using the direct image analysis as mentioned earlier.

In the experiment, a set of 48 samples with a desired volume of $47.83 \mathrm{~nL}\left(V_{\mathrm{d}}\right.$ in (3)) of the cubic phase (Monoolein/BCECF solution 60/40 (w/w)) is delivered. The cubic phase is delivered from 21 steps $(262.5 \mu \mathrm{m})$ above the base of the well with a 26 gauge $(0.26-\mathrm{mm}$ internal diameter) needle. To deliver the desired volume accurately, an initial prime volume serving as $V_{\mathrm{r}}$ is expelled for the first well as shown in Fig. 8. Here, $V_{\mathrm{r}}$ is $47.83 \mathrm{~nL}$. Then, every well is delivered with a volume of $V_{\mathrm{o}}$ which is $47.83 \mathrm{~nL}$. From (3), one can see that without $V_{\mathrm{r}}, V_{\mathrm{d}}$ will be less than $V_{\mathrm{o}}$. The result of the experiment is represented in Fig. 15 where the predicted volume of the cubic phase is calculated using the model proposed and shown as the solid line in the figure. The actual volume of the cubic phase dispensed in the 48 wells is represented by the dashed line in Fig. 15. From Fig. 15, one can observe that the actual volume of the dispensed cubic phase very much follows the predicted volume. On the set of 48 samples, the average difference between the actual volume delivered and model predicted volume is $3.78 \mathrm{~nL}(7.9 \%)$ while the maximum deviation and minimum deviation of the predicted volume from the actual volume in a set of 48 samples is $9.48 \mathrm{~nL}$ $(19.8 \%)$ and $0.11 \mathrm{~nL}(0.24 \%)$, respectively.

It is interesting to examine the goodness of the model by studying the result of the first well. As mentioned earlier, the initial prime volume of $47.83 \mathrm{~nL}$ is expelled out of the needle before the dispensing starts. Using the dispensing height, which is $262.5 \mu \mathrm{m}$, and the prime volume, one can calculate the radius of the ellipsoid to be $R=0.285 \mu \mathrm{m}$ using (1). When the cubic phase is delivered to the first well, an additional volume serving as $V_{\mathrm{O}}$, which is $47.83 \mathrm{~nL}$, is dispensed. Using (2), the delivered volume to the first well $V_{\mathrm{d}}$ should be $68.33 \mathrm{~nL}$. The actual volume delivered is $66.54 \mathrm{~nL}$, and the difference between that and the volume predicted by the model is $1.79 \mathrm{~nL}$. Without the model, one would expect that the entire $47.83 \mathrm{~nL} \times 2=95.66$ $\mathrm{nL}$ would be delivered for which the difference is $27.33 \mathrm{~nL}$.

Choosing $V_{\mathrm{r}}=47.83 \mathrm{~nL}$ is purely experimental; we could choose a smaller value. From the previous discussion, one can see that after the first dispense, $V_{\mathrm{r}}$ is $27.33 \mathrm{~nL}$, which serves well for the remaining wells. Unfortunately, the initial $V_{\mathrm{r}}$ is difficult to determine considering that so many factors are involved. Experimental results prove that letting $V_{\mathrm{r}}=V_{\mathrm{O}}$, where $V_{\mathrm{O}}$ is the desired volume of dispense, the actual volume delivered converges to the desired volume quickly. In this way, one can avoid repeated tests to choose an optimal $V_{\mathrm{r}}$ while the performance is still acceptable especially when many wells need to be dispensed.

\section{Verification of the Cubic-Phase Delivery Using Fluorescence}

For the purposes of calibration and verification, we have used an image-analysis method to determine the volume of the solution or the cubic phase in each well. The volume of the delivered material is obtained from the product of the calculated area and the well thickness. The approach is very convenient provided that the delivered volume is proportional to the area of the material. We consider this method to be reasonable because the well is very thin $(0.13 \mathrm{~mm})$, and the delivered material has a disc shape once the cover slip is in place. To verify this assumption and the validity of the method, a different technique was used to determine the volume of the delivered cubic-phase bolus. This approach will be described. 


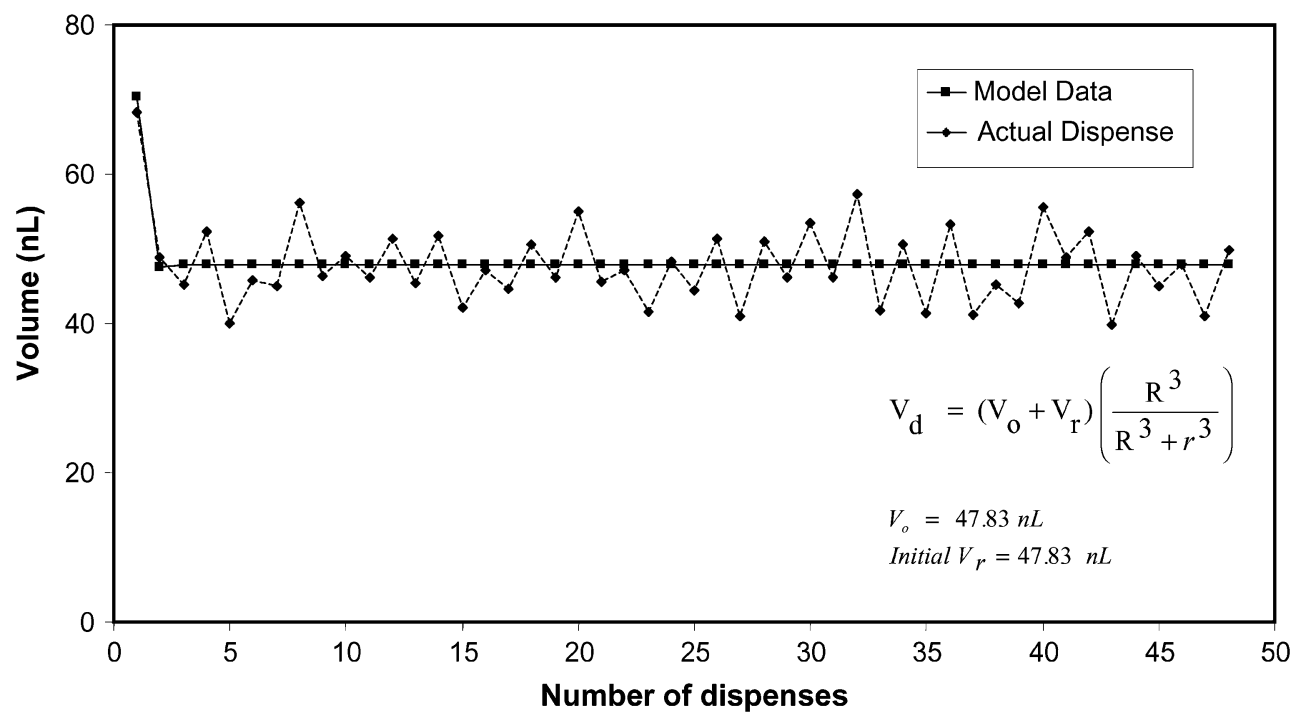

Fig. 15. Actual volume of the cubic phase delivered in comparison with predicted data when delivering $47.83 \mathrm{~nL}$ of the cubic phase (Monoolein/BCECF solution $60 / 40(\mathrm{w} / \mathrm{w})$ ) from 21 steps (approximately $0.2625 \mathrm{~mm}$ ) above the base of the well with a 26-gauge $(0.26$-mm internal diameter) needle and an initial prime volume of $47.83 \mathrm{~nL}$. Note (3) in the figure.

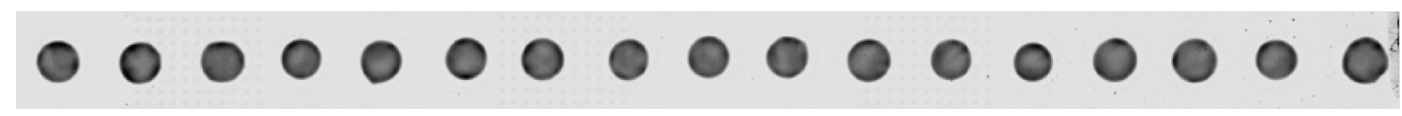

Fig. 16. Image of one row of 17 boluses obtained from measuring the fluorescence intensity.

The reproducibility of the system with which volumes of lipid/protein mesophase were delivered was assessed by a fluorescence intensity measurement using a sample that had been doped with a small amount of the highly fluorescent lipid, Texas Red-PE (1381.85 g/mole, lot 45B1-7, purchased from Molecular Probes, Inc., Eugene, OR). For this purpose, monoolein with 1.0 mole\% Texas Red-PE, was prepared gravimetrically. This was used as a stock solution and was then serially diluted to 0.0001 mole\% Texas Red-PE in monoolein. The mixtures were homogenized by mechanical mixing in the molten state at $40{ }^{\circ} \mathrm{C}$. The cubic phase containing $60 \% \mathrm{w} / \mathrm{w}$ of monoolein/Texas Red-PE and $40 \%$ w/w water was prepared using the lipid-mixing device.

For delivery quantification, 5 rows of 17 replicate boluses (a total of 85 individual boluses) of 47.83-nL doped-cubic phase were delivered onto standard $75 \times 25-\mathrm{mm}$ microscope glass slides at room temperature using the in meso robot. As mentioned earlier, the distance between the tip of the dispensing syringe needle and the glass plate surface must be adjusted to optimize delivery. After delivery of the cubic phase, the slide dried for $1 \mathrm{~h}$ at room humidity and then the lipid was melted at $40^{\circ} \mathrm{C}$.

Fluorescence intensity measurements were performed using an Affymetrix 428 scanner (excitation, $535 \mathrm{~nm}$; emission, 575 $\mathrm{nm}$; spatial resolution, $10 \mu \mathrm{m}$; gain $45 \mathrm{~dB}$ ). Scanned images were saved as 16-b tiff files and processed using the Fit2D program to generate plots of projected intensity versus distance along a row on the plate. Integrated peak areas were corrected for background between peaks, and Excel was used to calculate averages and coefficients of variance.

The desired delivered volumes $\left(V_{\mathrm{d}}\right)$ were arrived at by knowing the advance per step of the motorized pump driving the dispensing syringe plunger. The average percent deviation of the calculated intensities for the 85 boluses was seen to be $6.4 \%$, indicating that the actual dispense of a $47.83-\mathrm{nL}$ volume of the cubic phase was precise to within the errors indicated by the image analysis method (7.9\%). One row of 17 boluses dispensed is shown in Fig. 16.

\section{Crystallization Setups}

The robotic system is very flexible to set up crystallization trials using different techniques. The robotic system has been programmed and used for setting up in meso, bicelle, microbatch, and sitting drop crystallization trials. It currently takes approximately 13 min to complete the entire crystallization setup. The protein crystals grown by the robotic system using different crystallization techniques are shown in Fig. 17. Since the emphasis of this paper is to introduce the robotic system which enables the automatic handling of nanoliter volumes of highly viscous biomaterials involved in the bio-chemistry process and not the process itself, the detailed procedures for growing the protein crystals are not presented here. For the latter, interested readers are referred to the references mentioned in this paper.

\section{CONCLUSION}

A robotic system for crystallizing membrane and soluble proteins in lipidic mesophases has been developed. The unique ability of the robotic system is to deliver nanoliter volumes of highly viscous biomaterials. The system is relatively inexpensive and can set up crystallization trials in high-throughput mode with only $20 \mathrm{~nL}$ of protein and $30 \mathrm{~nL}$ of lipid per well.

The liquid handling parameters of the robotic system are scaled and optimized for accurate and reproducible delivery of 


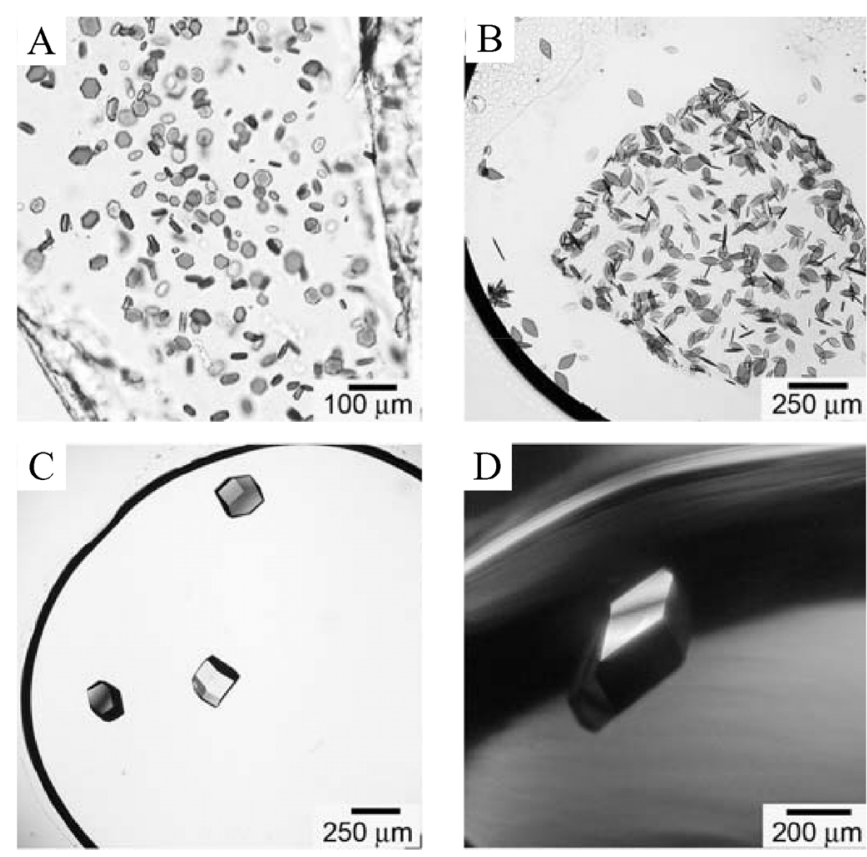

Fig. 17. (a) Crystals of bacteriorhodopsin grown by the in meso method after 5 days at $20^{\circ} \mathrm{C}$. (b) Crystals of bacteriorhodopsin grown by the bicelle method after 7 days at $37^{\circ} \mathrm{C}$. (c) Crystal of lysozyme grown by the microbatch method after 1 day at $20^{\circ} \mathrm{C}$. (d) Crystals of lysozyme grown by the sitting drop vapor diffusion method after 5 days at $20^{\circ} \mathrm{C}$.

microliter volumes of different precipitant solutions. The various factors that impact the delivery of nanoliter volumes of viscous materials are explained. An innovative cubic-phase-based coordinate measuring mechanism is developed which makes use of the conductivity of the cubic phase to achieve the resolution of $12.5 \mu \mathrm{m}$ in the measuring of the dispensing distance. A mathematical model to determine the actual volume dispensed and its variation with needle diameter, height from which it is dispensing, and desired volume to be delivered is proposed.

The system is validated by performing the quantitative analysis of the cubic phase and precipitant solution delivery. The coefficient of variance in delivering $50 \mathrm{~nL}$ of the cubic phase and microliter volumes of different precipitant solution is less than $10 \%$ by using the imaging analysis method. The actual difference as measured by the fluorescence measurement method is even less $(6.4 \%)$. The developed robotic system is quite versatile in setting up different crystallization trials.

The current robotic system delivers a disc-shaped bolus of cubic phase. In the future, we want to modify the motion profile of the dispensing tool to deliver different shapes of cubic phase, as the shape of the cubic phase can affect the precipitant solution diffusion that could influence protein crystallization. We are also working on picoliter delivery of viscous cubic phase for protein microarray fabrication applications.

\section{REFERENCES}

[1] V. Luzzati, "X-ray diffraction studies of lipid-water systems," in Biological Membranes, Physical Facts and Functions, D. Chapman, Ed.. Ithaca, NY: Academic, 1968, vol. 1, pp. 71-123.

[2] M. Caffrey, "X-ray diffraction as a technique studying the mesomorphic phase properties of lipids," in Membranes, Metabolism and Dry Organisms, A. Leopold, Ed.. Ithaca, NY: Cornell Univ. Press, 1986, pp. $350-357$.
[3] J. Luft, R. Collins, N. Fehrman, A. Lauricella, C. Veatch, and G. DeTitta, "A deliberate approach to screening for initial crystallization conditions of biological macromolecules," J. Struct. Biol., vol. 142, pp. $170-179,2003$.

[4] E. Landau and J. Rosenbusch, "Lipidic cubic phases - A novel concept for the crystallization of membrane proteins," in Proc. National Academy Science, 1996, vol. 93, pp. 14532-14535.

[5] M. Caffrey, "Membrane protein crystallization," J. Struct. Biol., vol. 142, pp. 108-132, 2003.

[6] H. Qiu and M. Caffrey, "Phase properties of the monoolein/water system: Metastability and equilibrium aspects," Biomaterials, vol. 21, pp. 223-234, 2000.

[7] S. Tokumoto, Y. Fujita, and S. Hirai, "Deformation modeling of viscoelastic objects for their shape control," in Proc. Int. Conf. Robotics and Automation, Detroit, MI, May 1999, pp. 767-771.

[8] S. Tokumoto, Y. Fujita, and S. Hirai, "Deformation transition graphs in forming operations of rheological objects," in Proc. Int. Conf. Robotics and Automation, San Francisco, CA, Apr. 2000, pp. 3071-3076.

[9] M. Chen and Y. F. Zheng, "Vibration-free handling of deformable beams by robot end-effectors," J. Robot. Syst., pp. 329-347, May 1995.

[10] B. Hannaford, J. Hewitt, T. Maneewarn, S. Venema, M. Appleby, and R. Ehresman, "Telerobotic remote handling of protein crystals," in Proc. Int. Conf. Robotics and Automation, Albuquerque, NM, Apr. 1997, pp. 1010-1015.

[11] R. C. Stevens, "High-throughput protein crystallization," Curr. Opin. Struct. Biol, vol. 10, pp. 558-563, 2000.

[12] T. Walter, J. Diprose, J. Brown, R. Pickford, R. Owens, D. Stuart, and K. Harlos, "A procedure for setting up high-throughput nano liter crystallization experiments, I. protocol design validation," J. Appl. Cryst, vol. 36, pp. 308-314, 2003.

[13] B. Rupp, B. W. Segelke, H. I. Krupka, T. Lekin, J. Schafer, A. Zemla, D. Toppani, G. Snell, and T. Earnest, "The TB structural genomics consortium crystallization facility: towards automation from protein to electron density," Acta. Cryst. D, vol. 58, pp. 1514-1518, 2002.

[14] D. Stock, O. Perisic, and J. Lowe, "Robotic nano liter protein crystallization at MRC laboratory of molecular biology," Progr. Biophys. Molec. Biol., vol. 88, pp. 311-327, 2005.

[15] U. Heinemann, K. Bussow, U. Mueller, and P. Umbach, "Facilities and methods for the high-throughput crystal structural analysis of human proteins," Acc. Chem. Res., vol. 36, pp. 157-163, 2003.

[16] D. Meldrum, C. Fisher, M. Moore, M. Saini, M. Holl, W. Pence, S. Moody, D. Cunningham, and P. Wiktor, "ACAPELLA-5K, a highthroughput automated genome and chemical analysis system," in Proc. IEEE/RSJ Int. Conf. Intelligent Robots and Systems, Las Vegas, NV, Oct. 2003, pp. 2321-2328.

[17] V. Cherezov and M. Caffrey, "Nano-volume plates with excellent optical properties for fast inexpensive crystallization screening of membrane proteins," J. Appl. Cryst, vol. 36, pp. 1372-1377, 2003.

[18] L. Muthusubramaniam, A. Peddi, Y. F. Zheng, V. Cherezov, and M. Caffrey, "Automating crystallization of membrane proteins by robot with soft coordinate measuring," in Proc. IEEE Int. Conf. Robotics and Automation, New Orleans, LA, Apr. 2004, pp. 1450-1455.

[19] A. Peddi, Y. F. Zheng, V. Cherezov, and M. Caffrey, "Automating the dispense of highly viscous biomaterials," in Proc. IEEE/RSJ Int. Conf. Intelligent Robots and Systems, Sendai, Japan, Sep. 2004, pp. $1281-1286$.

[20] V. Cherezov, A. Peddi, L. Muthusubramaniam, Y. F. Zheng, and M. Caffrey, "A robotic system for crystallizing membrane and soluble proteins in lipidic meso phases," Acta. Cryst. D, vol. D60, pp. 1795-1807, 2004.

[21] C. Hansen, E. Skordalakes, J. Berger, and S. Quake, "A robust and scalable microfluidic metering method that allows protein crystal growth by free interface diffusion," PNAS, vol. 99, pp. 16531-16536, Dec. 26, 2004.

[22] V. Cherezov and M. Caffrey, "A simple and inexpensive nanolitervolume dispenser for highly viscous materials used in membrane protein crystallization," J. Appl. Cryst., vol. 38, pp. 398-400, 2005.

[23] "Handbook of Chemistry and Physics," 87th ed. D. R. Lidle, Ed., 2006 CRC, Boca Raton, FL.

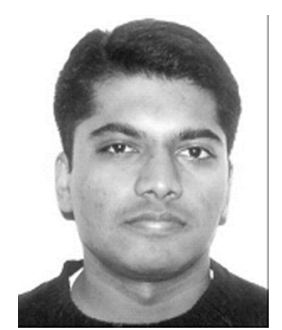

Avinash Peddi received the M.S. degree in electrical and computer engineering from The Ohio State University (OSU), Columbus, in 2005, and the B.E. degree in electronics and communication engineering from Osmania University, Hyderabady, India, in 2002.

He was with the Department of Electrical and Computer Engineering at OSU. Currently, he is an Integration Project Manager with Tecan US, Durham, NC. 


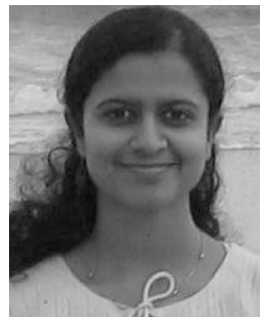

Lalitha Muthusubramaniam received the B.Sc. degree in electrical and electronics engineering from Madras University, Madras, India, and the M.Sc. degree in biomedical engineering from The Ohio State University, Columbus.

Her graduate research primarily focused on developing and building an automated system for membrane protein crystallization using the cubic-phase method. Her other responsibilities included characterization of lipid phase behavior and microstructure using X-ray diffraction techniques and setting up membrane protein crystallization trials on the robot in accordance with the phase behavior studies

She was a Staff Biochemist with Merck Research Laboratories (MRL), North Wales, PA, for three years where she performed screening of compounds for hit prioritization and lead identification. Her responsibilities included robotic integration of label-free technologies and development of assays to perform high-content screens in an automated and miniaturized fashion. She is currently with Genentech, South San Francisco, CA, where she continues to work on high throughput screening with the additional responsibility of helping with the management of the small molecule compound library at Genentech.

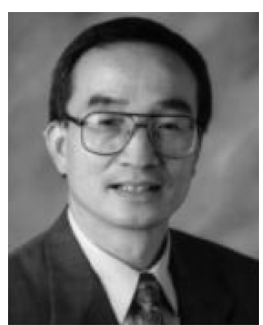

Yuan F. Zheng (F”97) received the M.S. and Ph.D degrees in electrical engineering from The Ohio State University (OSU), Columbus, in 1980 and 1984, respectively.

From 1984 to 1989 , he was with the Department of Electrical and Computer Engineering at Clemson University, Clemson, SC. Since 1989, he has been with The OSU, where he is currently Professor and was the Chairman of the Department of Electrical and Computer Engineering from 1993 to 2004. From 2004 to 2005 , he spent a sabbatical year at the Shanghai Jiao Tong University, Shanghai, China, and continues to be involved as Dean of School of Electronic, Information and Electrical Engineering for part-time administrative and research activities. His research interests include two aspects. One is in wavelet transform for image and video, and object classification and tracking, and the other is in robotics, which includes robotics for biological applications, multiple robots coordination, legged walking robots, human-robot coordination, and personal robotics.

Dr. Zheng was and is on the editorial board of five international journals.He received the Presidential Young Investigator Award from Ronald Reagan in 1986, and the Research Awards from the College of Engineering of The OSU in 1993 and 1997, respectively. He, along with his students, received the best student paper award a few times in 2000, 2002, and 2006, respectively, and received the Fred Diamond Award for best technical paper in 2006. In 2004, Prof. Zheng was appointed to the International Robotics Assessment Panel by the National Science Foundation.

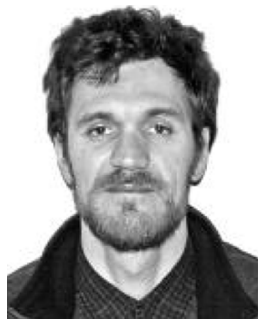

Vadim Cherezov received the M.S. and Ph.D. degrees from the Moscow Institute of Physics and Technology, Moscow, Russia.

He worked in the Frank Laboratory of Neutron Physics at the Joint Institute for Nuclear Research, Dubna, Russia, from 1992 to 1998. From 1998 to 2006, he was a Postoctoral Researcher and Research Associate with the Chemistry Department at The Ohio State University, Columbus. Currently, he is a Scientist at The Scripps Research Institute, La Jolla, studies of membrane proteins.

CA. His research interests focus on the structural

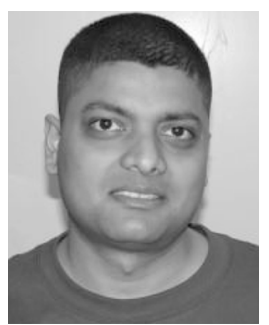

Yohann Misquitta received the Ph.D. degree in biophysics from The Ohio State University (OSU), Columbus.

During that time he was with the Department of Chemistry at OSU. Currently, he is a Postdoctoral Researcher at the School of Sciences, University of Limerick, Limerick, Ireland. His research interests are lipid-phase behavior in relation to membrane protein crystallization.

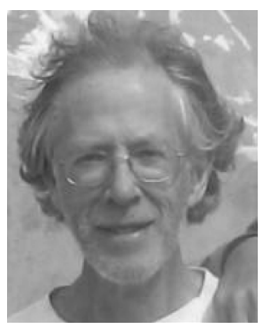

Martin Caffrey received the B.Agr.Sc. degree in agricultural chemistry from University College, Dublin, Ireland, in 1972, the M.S. degree in food science, and the Ph.D. degree in biochemistry from Cornell University, Ithaca, NY, in 1976 and 1982, respectively.

From 1982 to 1987, he was a Postdoctorate Research Associate in biochemistry at Cornell University. From 1988 to 2003, he was an Assistant, Associate, and Full Professor in the Department of Chemistry at The Ohio State University (OSU), Columbus. Currently, he is a Professor of membrane structural biology at the College of Science, University of Limerick, Limerick, Ireland, and Adjunct Professor in the Department of Chemistry at OSU. He was the Editor for the Membranes Issue of Current Opinions in Structural Biology in 2000 and Associate Editor of the Biophysical Journal from 1998 to 2002. He was a Lecturer for the NATO ASI School: Electrostatic Effects in Soft Matter and Biophysics, Les Houches, France, in 2000.

Dr. Caffrey has received many awards and honors for his research contributions including, in recent years, the Chaired Professorship at the University of Limerick in 2003, the Science Foundation Ireland Investigatorship Award in 2003, and the Outstanding International Faculty Award at OSU in 2002. He was Chair of the Small Angle Scattering-Applications Microsymposium of the XVII Congress, Seattle, WA, in 1996; Organizer of the V. Luzzati 75th Birthday International Symposium, Paris, France in 1998; and Organizer of the Databases for Biophysicists Workshop, Biophysical Society 45th Annual Meeting, Boston, MA, in 2001. He participated in the Macromolecular Crystallography course of the Cold Spring Harbor Laboratory (CSHL), Cold Spring Harbor, NY, in 1999. In 2006, he was an Instructor at the CSHL Macromolecular Crystallography course. He was Visiting Scientist at the French Atomic Energy Commission, Grenoble, France, in 1999, and Visiting Professor at the Joseph Fourier University, Grenoble, in 2000. 